\title{
An analysis of reporting quality of prospective studies examining community antibiotic use and resistance
}

\author{
Mina Bakhit ${ }^{*}$, Chris Del Mar, Anna Mae Scott and Tammy Hoffmann
}

\begin{abstract}
Background: Antibiotic resistance is a global problem, but the relationship between antibiotic use and resistance development and decay is not well understood. This knowledge is best provided by prospective studies, but to be useful they must be both conducted and reported well. Little is known about the reporting quality of these studies. This study aimed to assess the quality of reporting in prospective studies that investigated antibiotic resistance following antibiotic exposure in community-based individuals.

Methods: The quality of reporting of prospective studies (17 randomised trials, eight cohort studies) identified in a systematic review of the relationship between antibiotic use and resistance were assessed independently by two researchers using checklists (one for trials, one for cohort studies) developed from existing reporting guidelines for these designs and this field.

Results: The mean percentage (SD, minimum-maximum) of mandatory items that were adequately described by the included studies was $59 \%$ for trials $(14 \%, 36-84 \%)$ and $52 \%$ for cohort studies $(17 \%, 13-70 \%)$. Most studies adequately described the study background and rationale, the type, combination, and duration of the antibiotic intervention, and the sampling procedures followed to isolate resistant bacteria. Most studies did not report the incident numbers of resistant and susceptible isolates analysed at each time point. Blinding and sample size calculation was inadequately reported in almost half of the trials and all cohort studies.
\end{abstract}

Conclusions: The quality of reporting in prospective studies investigating the association between antibiotic exposure in the community and isolation of resistance isolates is variable. Some details were missing in more than half of the studies, which precludes a complete risk of bias assessment and accurate interpretation and synthesis of results.

Keywords: Antibiotic resistance, Quality of reporting, Primary care

\section{Background}

Antibiotic resistance is a global public health concern, threatening lives by jeopardising successful treatment of a vast range of bacterial infections [1,2]. It is estimated that 10 million people may die in 2050 because of resistance [3]. Antibiotic prescribing levels in primary care are high $[4,5]$, even though for many of the conditions for which they are prescribed (such as acute respiratory infections), antibiotics provide minimal benefits and these may not be outweighed by the harms of their use [6-10].

\footnotetext{
* Correspondence: mbakhit@bond.edu.au

Centre for Research in Evidence-Based Practice (CREBP), Faculty of Health Sciences and Medicine, Bond University, Robina, QLD 4229, Australia
}

Antibiotic use drives resistance [11-13] and there is some indication that resistance decays over time [13]. Knowledge about the association between antibiotic use, the development of resistance, and timeframes of potential decay is important for informing public health messages, antibiotic resistance campaigns, and clinician training. However, evidence syntheses investigating the relationship between antibiotic exposure and the development and decay of resistance have been limited to two systematic reviews that have included mostly studies with retrospective designs $[11,12]$. Understanding the association between antibiotic exposure and isolation of resistance bacteria is best informed by prospective study designs. Such designs offer better opportunities to control for confounding

(c) The Author(s). 2018 Open Access This article is distributed under the terms of the Creative Commons Attribution 4.0 International License (http://creativecommons.org/licenses/by/4.0/), which permits unrestricted use, distribution, and 
factors, including more precise timeframes of the duration between antibiotic exposure and isolation of resistance bacteria, which helps to avoid the uncertainty that is implicit in 'time-until' periods that are dictated by retrospective designs. As global concern about resistance increases, an increasing number of prospective studies investigating this issue are being conducted.

However, to provide interpretable evidence and enable complete risk of bias assessment, these studies need to be reported clearly and comprehensively. There are characteristics of studies that measure antibiotic exposure and resistance that are not adequately captured by existing reporting checklists and researchers may not have adequate awareness of these issues and guidance about how to report such studies. We are not aware of any studies that have examined the quality of reporting of prospective studies about antibiotic use and resistance. We aimed to assess the completeness of reporting of prospective primary studies that examined antibiotic use and resistance.

\section{Method}

\section{Selection of included studies}

Studies were included in this study if they had been identified as part of a recently published systematic review that assessed the extent of bacterial resistance in individuals caused by antibiotic use in primary care, and the rate of decay of resistance [13].

Full details of the systematic review search are available elsewhere [13]. Briefly, we searched PubMed, EMBASE, and the Cochrane Central Register of Controlled Trials from inception until the first week of May 2017, using MeSH terms, keywords, and forward and backward citation searches. We included randomised controlled trials and prospective cohort studies that compared antibiotic-exposed patients in the community against controls. Our outcome was the prevalence of resistance bacteria over time.

\section{Assessment of the quality of reporting of included studies}

For the present study, we developed two assessment checklists (one for trials and one for prospective cohort studies), based on existing reporting guidelines relevant to these study designs and this field. For trials, the relevant guideline was the Consolidated Standards of Reporting Trials (CONSORT) statement [14]; for cohort studies, the relevant guidelines were Strengthening the Reporting of Observational studies in Epidemiology (STROBE) [15] and its extension for optimising reporting of epidemiological studies in Antimicrobial Stewardship (STROBE-AMS) [16]. Additional reporting recommendations that are relevant to both study types include the Template for Intervention Description and Replication (TIDieR
[17]) items and those specific to resistance reporting in systematic reviews of antibiotic interventions [18].

Informed by our experience from completing the systematic review, several items necessary for the accurate interpretation of results were added to the checklists. The resultant checklists were piloted with four epidemiologists and four antibiotic resistance researchers who used them to assess the quality of reporting of a small number of eligible studies. Following this, minor modifications were made to the grouping of the items, and the explanatory wording of some of the additional items. The final version of trial checklist had 89 items and the cohort checklist had 81 items.

Two researchers (MB and AMS) then used the checklists to independently assess the quality of reporting of each included study. Each item was rated 'Yes' (if the study adequately described the item), 'No' (if it did not), or 'Not applicable'. Agreement between assessors was reached through discussion after small batches of five studies were rated, and discrepancies resolved through discussion with a third researcher (TH or CDM). We did not calculate the agreement between the two assessors. The complete checklists with item descriptions and the source of each item are available in Additional files 1 and 2 .

\section{Data analysis}

Data were analysed using Microsoft Excel $^{\circledR} 2016$ (Microsoft, Redmond, WA, USA) and descriptive statistics were calculated.

\section{Results}

The sample of articles consisted of 17 randomised controlled trials and eight prospective cohort studies. The trials primarily assessed the risk of isolation of post-treatment carriage of resistant bacteria. They were published from 1982 to 2016 and conducted in ten countries. The cohort studies assessed changes in resistance patterns before and after antibiotic use. They were published from 1988 to 2008 and from seven countries (the full list of included articles is shown in Additional file 3).

\section{Completeness of reporting - trials}

For the 17 trials, 70 mandatory items and an additional 19 'if applicable' items were scored. Twelve (17\%) mandatory items were reported by all trials; one item (describing other organisms susceptible to the exposed antimicrobial or same class) was not reported by any trials. The mean percentage ( $\mathrm{SD}$, minimum-maximum) of the mandatory items that were adequately described by the trials was $59 \%(14 \%, 36-84 \%)$ (see Fig. 1). Additional file 4 shows the percentages of trials that adequately described each item including the 'if applicable' 
Were the following items described? Background and rationale Specific objectives or hypothesis

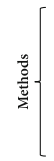

$$
\text { Study design }
$$

Description of the setting and location

Periods of recruitment Duration of follow-up

Characteristics of population served by the healthcare setting

participant selection methods
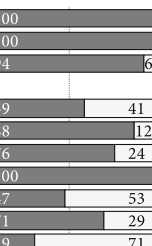

Type and combinations of antimicrobials 10 Dose provided and defined daily dosage (DDDs) 100 When was the antimicrobial treatment administered? Duration of exposure Route of administration

Who administered the Intervention fidelity - planned

Comparator (if antimicrobial treatment; type and combination

Defined outcome measures Definition of resistance, multidrug resistance, and co-resistance used Guidel ines used in laboratory resistance measurements<smiles>C1CC(C2CC2)C1</smiles>

Number of samples per person Sampling period Method of sampling Transport of samples

营

How was sample size determined?

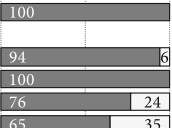

100
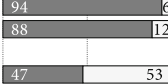

[

Method used to generate the random allocation sequence Type of randomisation Mechanism used to implement the random allocation sequence Steps taken to conceal the sequence Who gented the random allocition sequence

$$
\begin{array}{r}
\text { Who generated the random allocation sequence } \\
\text { Who enrolled participants }
\end{array}
$$
Who assigned participants to interventions

Who was blinded after assignment to interventions? Were microbiologists blinded to the time of sampling?

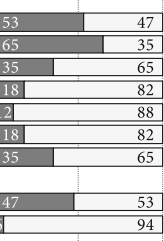

Unit of analysis defined 旁[

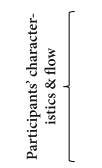

Characteristics of study participants Baseline resistance of the index pathogen Time since last antimicrobial exposure Time since last antimicrobial exposure 8 No. of participants who were randomly assigned, received treatment, and analysed Losses and exclusions after randomisation (with reasons) Intervention fidelity/adherence - actual

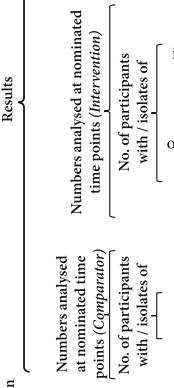

Total no. of participants with/isolates of the index pathogen No. of participants with sterile swabs

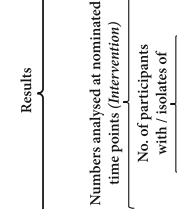
dex pathogen susceptible to the exposed antimicrobial or same class index pathogen resistant to the exposed antimicrobial or same class 88 index pathogen susceptible to other antimicrobial or different class 24 index pathogen resistant to other antimicrobial or different class er organisms susceptible to the exposed antimicrobial or same class other organisms resistant to the exposed antimicrobial or same class Total no. of participants with/isolates of other organisms No. of adverse event
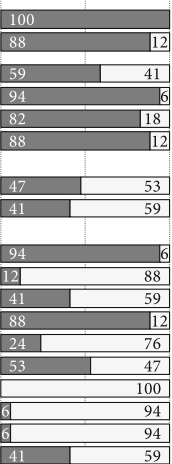

Total no. of participants with/isolates of the index pathogen Total no. of participants with/solticipants with sterile swabs
No. of participat dex pathogen susceptible to other antimicrobial or different class index pathogen resistant to other antimicrobial or different class Total no. of participants with/isolates of other organisms

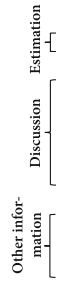

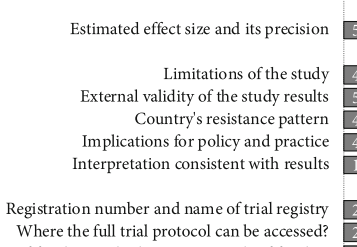

$$
\begin{aligned}
& \text { Limitations of the study } \\
& \text { External validity of the study results } \\
& \text { Country's resistance pattern }
\end{aligned}
$$$$
\text { Country's resistance pattern }
$$
Implications for policy and practice Interpretation consistent with results

Registration number and name of trial registry the full trial protocol can be accessed?
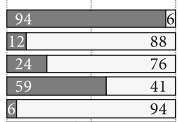

起 Sources of funding and other support, role of funders

Fig. 1 Quality of reporting, percentage of RCTs meeting each item (studies $=17$, mandatory items $=70$ ). $R C T$ randomised comtrolled trial 
items and Additional file 5 shows the percentage of items adequately described by each trial.

The items that were most commonly reported include those about: study background (all trials provided the study rationale and described any previous in vivo and/ or in vitro studies); intervention (most trials adequately described the type of antibiotics, duration and dose); and sampling (almost all trials adequately reported the sampling procedures followed for isolating resistant isolates including the site, number of samples per person, and sampling period).

Items that were poorly reported by many trials include those describing the sample size, randomisation, blinding, and the results. Almost half of the trials reported the sample size determination, and more than half of the trials poorly described the methods used for randomisation and allocation (including the person(s) responsible for generating the random allocation sequence, and the steps taken to conceal the randomisation sequence). Blinding reporting was incomplete in almost half of the trials and only one study reported blinding of microbiologists to the time of sampling. Many items describing the results were missing in the majority of the trials, including key details such as: the numbers analysed at nominated time points, particularly the number of isolates susceptible to the intervention or comparator (if applicable); and the number of participants with sterile swabs (clean-catch swabs) or resistance/susceptibility among other organisms (other than the index pathogen) isolated from other body site (other than the system/site of interest).

\section{Completeness of reporting - cohort studies}

In the eight cohort studies, 63 mandatory items and an additional 19 'if applicable' items were scored: seven (11\%) mandatory items were reported by all cohort studies; eight (13\%) were not reported by any. The mean percentage (SD, minimum-maximum) of the mandatory items that were adequately described by the studies was 52\% (17\%, 13-70\%) (see Fig. 2). Additional file 6 shows the percentage of cohort studies that adequately described each item including the 'if applicable' items and Additional file 7 shows the percentage of items adequately described by each study.

The items most commonly reported were those about the: study background (most described specific objectives and study rationale); outcome measures (most defined each measure and reported when each was measured); and sampling (most studies adequately described the sampling procedures used, including the site, number of samples per person, and sampling period).

Items that were poorly reported were those describing the sample size, measurement and results. None of the studies reported how their sample size was determined and none reported if resistance was measured by an independent laboratory or if the microbiologists were blinded. As with the trials, many key details about the numbers analysed were missing, such as the number of susceptible isolates to antibiotic exposure analysed at each of the nominated time points, along with the resistance/susceptibility among other organisms isolated from another body site.

\section{Discussion}

The quality of reporting of prospective studies examining antibiotic use and resistance varied in this study. Some aspects of the studies (such as the sampling procedures used and rationale for the study) were described in most, but some details were missing in many studies. Some of the missing items, such as those about blinding or the numbers analysed, are particularly important for assessing a study's risk of bias and interpreting its results accurately.

Few studies reported the incident numbers of both resistant and susceptible isolates analysed at each time point (and for both the intervention and/or comparator groups), and only one study reported the isolation of resistant isolates from body sites other than the target site/ system. This may be as important to know as the resistance in the originally infected site, from the point of view of antibiotic resistance generation in the microbiome, and hence in the community at large. Similarly, most studies only reported resistance to the class of antibiotic used, although some studies also reported resistance to other antibiotic classes - a possibly important omission because of induced co-resistance to antibiotics from different classes [19].

Reporting of how and whether blinding occurred was inadequate in almost half of the studies. Only one study reported whether the microbiologist was blinded to the time of sampling. Data on changes in resistance over time could be biased if those responsible for measuring resistance are not blinded to the time of sampling. As with other aspects of methods reporting, if a study does not describe a process, a reader cannot be sure if the process did not occur or was just not reported. This uncertainty impedes risk of bias assessment and decreases the confidence in the reported results.

While describing a study with sufficient detail to enable replication and interpretation of results is good scientific practice, many authors are not aware of all the details that need to be reported to sufficiently describe a study. To assist authors with comprehensively describing studies, reporting guidelines have been developed. However, for our sample of included studies, the impact of reporting guidelines would have been minimal, as most (88\%) of the included cohort studies were published before the release of the STROBE reporting guidelines 


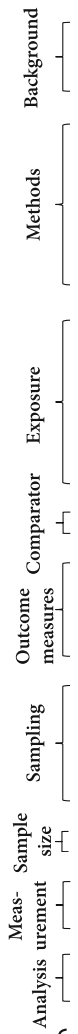

击

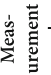

忿

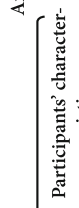

Background and rationale Previous clinical in vivo and/or in vitro studies Specific objectives or hypothesis

Study design Description of the setting and location Periods of recruitment Duration of follow-up Characteristics of population served by the healthcare setting Eligibility
Participant selection methods

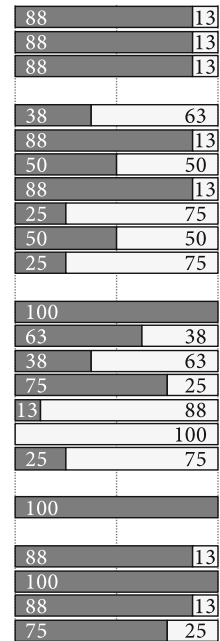
Defined outcome measures
When was each outcome measured?
Definition of resistance, multidrug resistance, and co-resistance used
Guidelines used in laboratory resistance measurements Defined outcome measures
When was each outcome measured?
Definition of resistance, multidrug resistance, and co-resistance used
Guidelines used in laboratory resistance measurements Defined outcome measures
When was each outcome measured?
Definition of resistance, multidrug resistance, and co-resistance used
Guidelines used in laboratory resistance measurements

Dose provided and defined daily dosage (DDDs) When was the antimicrobial treatment administered? Duration of exposure Route of administration

Who administered the antimicrobial treatment? Intervention fidelity - planned Comparator (if antimicrobial treatment; type and combinations) Number of samples per person Sampling period Method of sampling Method of sampling
Transport of samples

How was sample size determined?

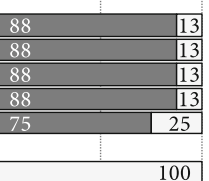

Was resistance measured by an independent laboratory? Were microbiologists blinded to the time of sampling?

Unit of analysis defined Statistical methods

Characteristics of study participants Baseline resistance of the index pathogen

Time since last antimicrobial exposure Description of potential confounders Information on exposures Indicate number of participants with missing data for each variable of interest Summary of follow-up time Intervention fidelity/adherence - actual

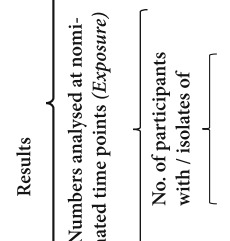

Total no. of participants with/isolates of the index pathogen
No of participants with sterile swabs index pathogen susceptible to the exposed antimicrobial or same class index pathogen resistant to the exposed antimicrobial or same class index pathogen susceptible to other antimicrobial or different class index pathogen resistant to other antimicrobial or different class other organisms susceptible to the exposed antimicrobial or same class other organisms resistant to the exposed antimicrobial or same class Total no. of participants with/isolates of other organisms No. of adverse events
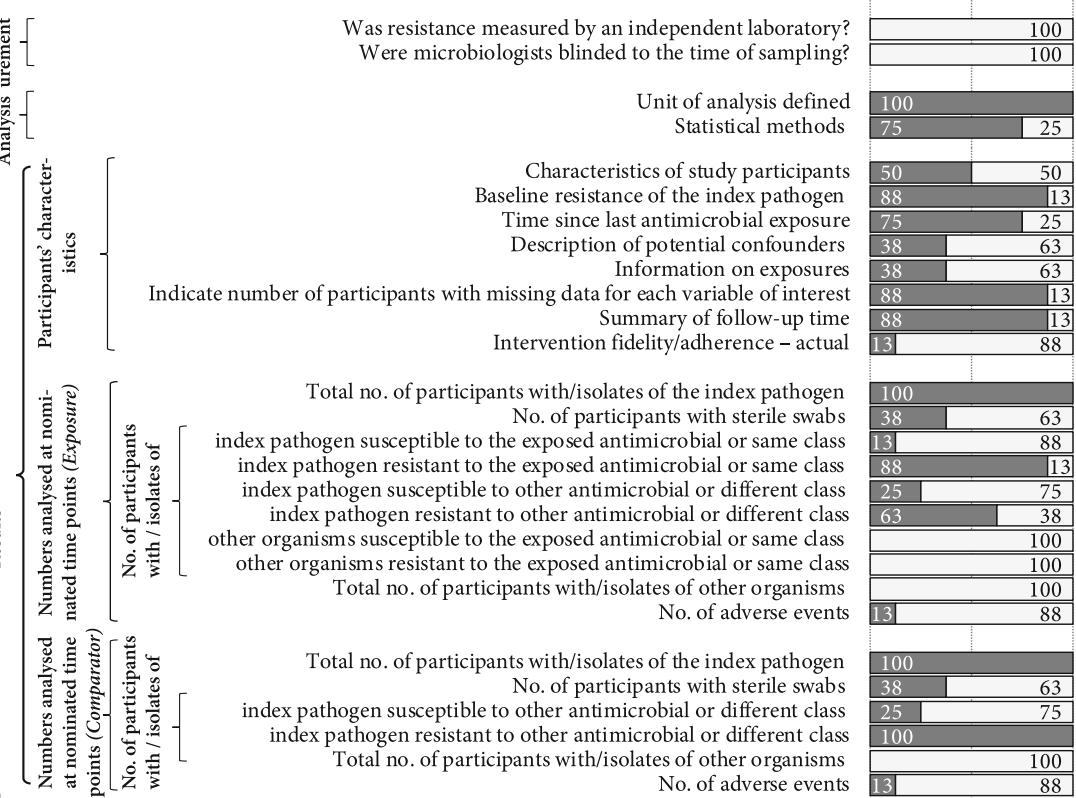

Total no. of participants with/isolates of the index pathogen No. of participants with sterile swabs index pathogen susceptible to other antimicrobial or different class index pathogen resistant to other antimicrobial or different class Total no. of participants with/isolates of other organisms

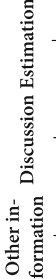

$$
\text { No. of adverse events }
$$

Estimated effect size and its precision

Limitations of the study External validity of the study results Country's resistance pattern Implications for policy and practice Interpretation consistent with results

Sources of funding and other support, role of funders

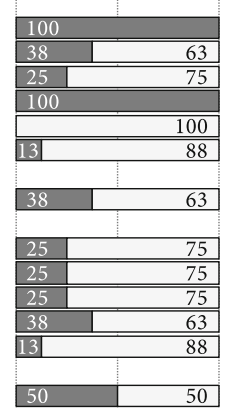

$\square \%$ of cohort studies that adequately described the item

$\square \%$ of cohort studies that did not adequately describe the item

Fig. 2 Quality of reporting, percentage of cohort studies meeting each item (studies $=8$, mandatory items $=63$ ) 
(and all before the STROBE-AMS publication), and all but one of the included trials were published before the 2010 CONSORT statement, although most (except three) were published before the 1996 CONSORT statement [20].

As our sample of studies is limited to those included in a systematic review of antibiotic resistance in individuals who were prescribed antibiotics in primary care, this may limit the generalisability of results beyond this setting. Additionally, the checklists used to assess the studies were modified from existing checklists and informed by the pragmatic experience of researchers assessing and synthesising these types of studies - the modified checklist have not been formally assessed. However, a strength of this study is the independent assessment of the included studies by two authors.

\section{Conclusions}

In this study of the reporting quality of prospective studies examining antibiotic use and resistance, just over half of the mandatory checklist items were adequately described for the randomised trials and cohort studies included. Some items (such as the type, combination, and duration of the antibiotic intervention, and the sampling procedures used to isolate resistant bacteria) were adequately described by most studies, whereas other details (such as the incident numbers of resistant and susceptible isolates analysed at each time point) were not described by most. Improving the quality of reporting of future studies that measure antibiotic resistance is necessary to aid accurate synthesis and interpretation of results. Better reporting may be facilitated by a reporting checklist, which is created following the recommendations for developing reporting guidelines [21], that is, specific to prospective studies of antibiotic use and resistance. This will help to improve the quality of reporting available to the research community, clinicians, and policy makers.

\section{Additional files}

Additional file 1: Checklist used to assess the RCTs and source of each item. (PDF $54 \mathrm{~kb}$ )

Additional file 2: Checklist used to assess the cohort studies and source of each item. (PDF $51 \mathrm{~kb}$ )

Additional file 3: List of studies included in the systematic review and assessed for their quality. (PDF $122 \mathrm{~kb}$ )

Additional file 4: Quality of reporting, percentage of RCTs meeting each item including the 'if applicable' items. (PDF $128 \mathrm{~kb}$ )

Additional file 5: Quality of reporting, percentage of items described by each trial. (PDF $29 \mathrm{~kb}$ )

Additional file 6: Quality of reporting, percentage of cohort studies meeting each item including the 'if applicable' items. (PDF $127 \mathrm{~kb}$ )

Additional file 7: Quality of reporting, percentage of items described by each cohort study. (PDF $31 \mathrm{~kb}$ )

\section{Abbreviations}

AMS: Antimicrobial stewardship; CONSORT: Consolidated Standards of Reporting Trials statement; STROBE: Strengthening the Reporting of Observational studies in Epidemiology; TIDieR: Template for Intervention Description and Replication

\section{Acknowledgements}

We kindly thank our colleagues who took part in piloting the checklists.

\section{Funding}

Funding for a PhD scholarship for the lead author was provided by the Australian National Health and Medical Research Council (\#1044904), which had no role in study design, data collection, data analysis, data interpretation, or writing of the report.

\section{Availability of data and materials}

The datasets used and/or analysed during the current study are available from the corresponding author on reasonable request.

\section{Authors' contributions}

$M B, C D M$ and $T H$ designed the study. MB and AMS performed data extraction and quality assessment. MB analysed the data and designed the figures. MB drafted the original manuscript and AMS, TH and CDM contributed to writing and revising the manuscript. All authors revised and approved the final manuscript.

Ethics approval and consent to participate Not applicable.

\section{Consent for publication}

Not applicable.

\section{Competing interests}

The authors declare that they have no competing interests.

\section{Publisher's Note}

Springer Nature remains neutral with regard to jurisdictional claims in published maps and institutional affiliations.

Received: 20 May 2018 Accepted: 3 November 2018

Published online: 27 November 2018

\section{References}

1. WHO. Antimicrobial resistance global report on surveillance. Geneva: World Health Organizetion; 2014.

2. WHO. Global antimicrobial resistance surveillance system (GLASS) report: early implementation 2016-2017. Geneva: World Health Organization. p. 2017.

3. O'Neill J. Antibiotic resistance: tackling drug-resistant Infections globally: final report and recommendations; 2016.

4. Del Mar C, Glasziou P, Lowe JB, van Driel ML, Hoffmann T, Beller E: Addressing antibiotic resistance - focusing on acute respiratory infections in primary care. Aust Fam Physician 2012, 41(11):839-840.

5. Gulliford MC, Dregan A, Moore MV, Ashworth M, Staa T, McCann G, Charlton J, Yardley L, Little P, McDermott L. Continued high rates of antibiotic prescribing to adults with respiratory tract infection: survey of 568 UK general practices. BMJ Open. 2014;4(10):e006245.

6. Ahovuo-Saloranta A, Rautakorpi UM, Borisenko OV, Liira H, Williams JW Jr, Makela M. Antibiotics for acute maxillary sinusitis in adults. Cochrane Database Syst Rev, 2014:2(2):CD000243.

7. Kenealy T, Arroll B. Antibiotics for the common cold and acute purulent rhinitis. Cochrane Database Syst Rev. 2013;6(6):CD000247.

8. Smith SM, Fahey T, Smucny J, Becker LA. Antibiotics for acute bronchitis. Cochrane Database Syst Rev. 2014;3(3):CD000245.

9. Spinks A, Glasziou PP, Del Mar CB. Antibiotics for sore throat. Cochrane Database Syst Rev. 2013;11(11):CD000023.

10. Venekamp RP, Sanders S, Glasziou PP, Del Mar CB, Rovers MM. Antibiotics for acute otitis media in children. Cochrane Database Syst Rev. 2013;1(1): CD000219.

11. Bell BG, Schellevis F, Stobberingh E, Goossens H, Pringle M. A systematic review and meta-analysis of the effects of antibiotic consumption on antibiotic resistance. BMC Infect Dis. 2014;14:13. 
12. Costelloe C, Metcalfe C, Lovering A, Mant D, Hay AD. Effect of antibiotic prescribing in primary care on antimicrobial resistance in individual patients: systematic review and meta-analysis. BMJ. 2010;340:c2096.

13. Bakhit M, Hoffmann T, Scott AM, Beller E, Rathbone J, Del Mar C. Resistance decay in individuals after antibiotic exposure in primary care: a systematic review and meta-analysis. BMC Med. 2018;16(1):126.

14. Schulz KF, Altman DG, Moher D, Group C. CONSORT 2010 statement: updated guidelines for reporting parallel group randomised trials. BMJ. 2010;340:с332

15. von Elm E, Altman DG, Egger M, Pocock SJ, Gøtzsche PC, Vandenbroucke JP. The Strengthening the Reporting of Observational Studies in Epidemiology (STROBE) statement: guidelines for reporting observational studies. Lancet. 2007;370(9596):1453-7.

16. Tacconelli E, Cataldo MA, Paul M, Leibovici L, Kluytmans J, Schroder W, Foschi F, De Angelis G, De Waure C, Cadeddu C, et al. STROBE-AMS: recommendations to optimise reporting of epidemiological studies on antimicrobial resistance and informing improvement in antimicrobial stewardship. BMJ Open. 2016;6(2):e010134.

17. Hoffmann TC, Glasziou PP, Boutron I, Milne R, Perera R, Moher D, Altman DG, Barbour V, Macdonald H, Johnston M, et al. Better reporting of interventions: template for intervention description and replication (TIDieR) checklist and guide. BMJ. 2014;348:g1687.

18. Leibovici L, Paul M, Garner P, Sinclair DJ, Afshari A, Pace NL, Cullum N, Williams HC, Smyth A, Skoetz N, et al. Addressing resistance to antibiotics in systematic reviews of antibiotic interventions. J Antimicrob Chemother. 2016;71(9):2367-9.

19. Carlet J, Jarlier V, Harbarth S, Voss A, Goossens H, Pittet D, Participants of the 3rd World Healthcare-Associated Infections F. Ready for a world without antibiotics? The Pensieres Antibiotic Resistance Call to Action. Antimicrob Resist Infect Control. 2012;1(1):11.

20. Begg C, Cho M, Eastwood S, Horton R, Moher D, Olkin I, Pitkin R, Rennie D, Schulz KF, Simel D, et al. Improving the quality of reporting of randomized controlled trials. The CONSORT statement. JAMA. 1996;276(8):637-9.

21. Moher D, Schulz KF, Simera I, Altman DG. Guidance for developers of health research reporting guidelines. PLoS Med. 2010;7(2):e1000217.

Ready to submit your research? Choose BMC and benefit from:

- fast, convenient online submission

- thorough peer review by experienced researchers in your field

- rapid publication on acceptance

- support for research data, including large and complex data types

- gold Open Access which fosters wider collaboration and increased citations

- maximum visibility for your research: over $100 \mathrm{M}$ website views per year

At BMC, research is always in progress.

Learn more biomedcentral.com/submissions 